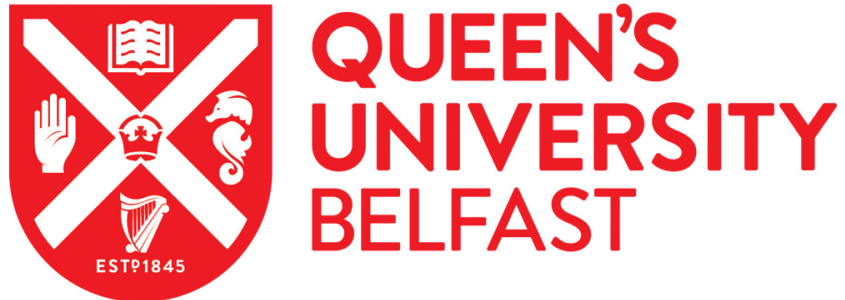

\section{Comparative study on the kinematic and static performance of two 1T2R parallel manipulators}

Lian, B., Chanal, H., \& Jin, Y. (2015). Comparative study on the kinematic and static performance of two 1T2R parallel manipulators. Paper presented at The 3rd IFtoMM Symposium on mechanism design for robotics, Aalborg, Denmark. http://www.meder2015.aau.dk/

\section{Document Version:}

Early version, also known as pre-print

Queen's University Belfast - Research Portal:

Link to publication record in Queen's University Belfast Research Portal

Publisher rights

(C) 2015 The Author(s)

\section{General rights}

Copyright for the publications made accessible via the Queen's University Belfast Research Portal is retained by the author(s) and / or other copyright owners and it is a condition of accessing these publications that users recognise and abide by the legal requirements associated with these rights.

Take down policy

The Research Portal is Queen's institutional repository that provides access to Queen's research output. Every effort has been made to ensure that content in the Research Portal does not infringe any person's rights, or applicable UK laws. If you discover content in the Research Portal that you believe breaches copyright or violates any law, please contact openaccess@qub.ac.uk. 


\title{
Comparative study on the kinematic and static performance of two $1 T 2 R$ parallel manipulators
}

\author{
Binbin Lian ${ }^{1,2}$, Hélène Chanal $^{3}$, Yan Jin ${ }^{1}$ \\ ${ }^{1}$ School of Mechanical and Aerospace Engineering, Queen's University Belfast, \\ UK, email:b.lian@qub.ac.uk;y.jin@qub.ac.uk \\ ${ }^{2}$ School of Mechanical Engineering, Tianjin University, China \\ ${ }^{3}$ Institut Pascal, Clermont Université, France, email: helene.chanal@ifma.fr
}

\begin{abstract}
Focusing on the potential of one translational and two rotational (1T2R) parallel kinematic machines (PKMs) for high precision manufacturing, this paper carried out the first time a comparative study on the kinematic and static performance of two promising Exechon variants, named PAW and PAW-II. PAW has the topology with 2RPU-SPR while the topology of PAW-II is 2RPU-R $\underline{P}$. Herein, R, $\mathrm{U}, \underline{\mathrm{P}}, \mathrm{S}$ denote revolute joint, universal joint, actuated prismatic joint and spherical joint, respectively. After introducing their architectures and inverse kinematics, two key performance characteristics, i.e. workspace and stiffness were conducted for the two PKMs. Comparative results show that PAW has a smaller workspace but better stiffness performance comparing to PAW-II.
\end{abstract}

Key words: parallel manipulators, parallel kinematic machine, workspace analysis, stiffness modelling

\section{Introduction}

In the past three decades, parallel kinematic machines (PKMs) have become a research hotspot among academia and industry, because of their higher stiffness, higher accuracy and higher dynamic capability comparing to serial manipulators. In the fields like aerospace manufacture and assembly, PKMs show huge potential as machine tools to meet the requirements of high precision, high working load and high productivity [1]. Equipped with three degree-of-freedom (3-DoF) PKMs which provide one translational and two rotational (1T2R) motion DoFs, 5-axis hybrid manipulators such as Tricept mechanism [2,3] and Sprint Z3 head [4, 5] are successful applications of these PKMs.

Although a large number of 3-DOF PKMs have been produced [6, 7], the PKMs which can offer both translational and rotational DOFs have not been extensively studied. In particular, the PKMs with 1T2R DOFs have special importance in machine tools as they can possibly replace the existing problematic two serial rotational motion axes which bring negative effects on the response 
speed and stiffness of 5-axis machine tools. Among these existing 1T2R PKMs, axial symmetrical 3 RPS architecture and its variant $3 \underline{P R S}$ architecture are the main focus. Research has been conducted on their kinematic analysis and optimization [8, 9, 10, 11], dynamic performance [12, 13], accuracy [14] and stiffness [15]. It is obvious that 3RPS PKM and $3 \underline{P R S}$ PKM are constructed by the same set of kinematic joints but of different topology in each leg. Despite lots of work has been conducted on these two PKMs independently, there is no special focus on the effects of different joint arrangements to the performance of the whole mechanism, which is one of the important issues in designing PKMs.

Similar to the concept of 3PRS PKM, Neumann [16] invented a promising machine tool called Exechon which consists of a novel 1T2R PKM and a 2-DOF serial wrist. The unique over-constrained architecture enables Exechon to achieve rather high stiffness and accuracy [17]. Inspired by the Exechon PKM, its variants PAW and PAW-II are recently proposed [18]. PAW has a 2RPU-SPR architecture and PAW-II has a 2RPU-R $\underline{P S}$ architecture. According to the mobility analysis, both PAW and PAW-II PKMs have 1T2R motion DOF and over-constrained architectures which led to simpler mechanisms with less number of links and joints [18]. It is believed that these two variants of Exechon are especially suitable for aerospace manufacturing tasks along curved surface. In order to further understand their performances, this paper carries out the comparative study of the two novel PKMs. Specially, as the only disparity of PAW and PAW-II is from the joint sequence of the leg, the comparative study will also shed light to the effect of varied joint arrangement, which will provide insightful knowledge in PKM design.

Having outlined the challenges mentioned above, the reminder of the paper is organized as follows. Section 2 describes the architecture of PAW and PAW-II and establishes their inverse kinematics. In Section 3, the orientation workspaces of the two PKMs are investigated with the same set of geometrical parameters, while their end-effector deflections with applied efforts are formulated by strain energy and Castigliano's theorem in Section 4. The conclusions are drawn in Section 5.

\section{Architecture description and inverse kinematics}

As shown in Fig. 1(a), PAW consists of fixed base, moving platform and three legs in between. $\operatorname{Leg}_{1}$ and $\operatorname{leg}_{3}$ are identical. Each of them has a RPU chain, with an R joint connected to the fixed base, linked to a linear actuator and a $U$ joint to the moving platform. The two axes connected to the moving platform of the two $U$ joints from $\operatorname{leg}_{1}$ and $\operatorname{leg}_{3}$ are collinear, while the other two axes of the two $U$ joints are in parallel with the $R$ joints on the base of the two legs. The topology of $\operatorname{leg}_{2}$ is $\mathrm{SPR}$ and the $\mathrm{R}$ joint axis is parallel to the collinear axis of $\mathrm{U}$ joints. The three legs are all actuated by linear actuators. Fig 1(b) shows the architecture of PAW-II whose $\operatorname{leg}_{2}$ is slightly different from PAW. Leg $_{2}$ of PAW-II is composed of one R joint on the base, followed by one linear actuator and one $S$ joint connecting to the moving platform. 
For the convenience to describe the motions, the same coordinate systems are defined for PAW and PAW-II. $A_{i}$ and $B_{i}(i=1,2,3)$ denote the joint centers to the base and moving platform respectively. The distance between point $A_{1}\left(B_{1}\right)$ to point $A_{3}\left(B_{3}\right)$ is $l_{1}\left(l_{3}\right)$. Point $O$ is the middle point of $A_{1} A_{3}$ and the base coordinate system $\left\{O-X_{b} Y_{b} Z_{b}\right\}$ is assigned to it, in which $X_{b}$ is directed from point $A_{1}$ to point $A_{3}, Y_{b}$ is from point $O$ towards point $A_{2}$. Similarly, point $O_{e}$ is the central point of $B_{1} B_{3}$. The platform coordinate system $\left\{O_{e}-X_{e} Y_{e} Z_{e}\right\}$ is defined at point $O_{e}$, and $X_{e}$ is directed from point $B_{1}$ to point $B_{3}, Y_{e}$ from point $O_{e}$ to point $B_{2}$. The distance between point $O\left(O_{e}\right)$ to point $A_{2}\left(B_{2}\right)$ is $l_{2}$ $\left(l_{4}\right)$.

Mobility analysis shows that both PAW and PAW-II have 1T2R DoF [18]. The posture of moving platforms can be expressed by three parameters $\alpha, \beta$ and $z_{e}$ (See Fig. 2). $\alpha$ represents the angle of frame $\left\{O_{e}\right\}$ rotating about $Y_{b}$ measured from $X_{b}$ to $X_{e}, \beta$ denotes the angle between the platform and the plane formed by points $A_{1}, B_{1}, B_{3}$ and $A_{3}$. Let ${ }_{o}^{o} R$ be the rotation matrix of frame $\left\{O_{e}\right\}$ to frame $\{O\}$, and it is formulated as

$$
{ }_{O_{e}}^{o} R=\left[\begin{array}{ccc}
c \alpha & -c \beta \cdot s \alpha & -s \beta \cdot s \alpha \\
0 & s \beta & -c \beta \\
s \alpha & c \beta \cdot c \alpha & s \beta c \alpha
\end{array}\right]
$$

where $s$ represents the sine function, and $c$ the cosine function.

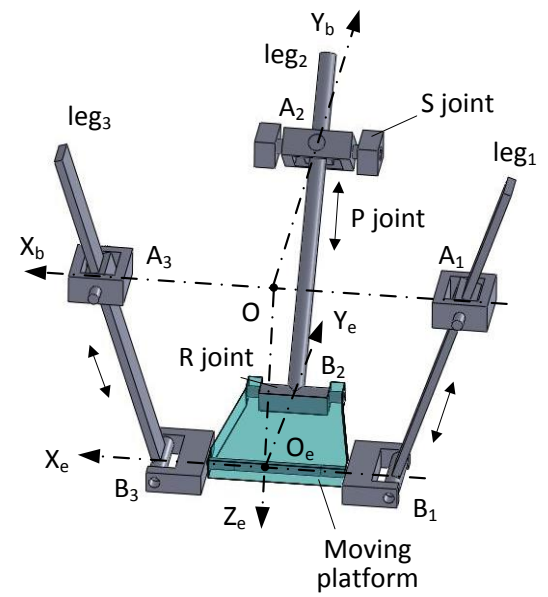

(a)

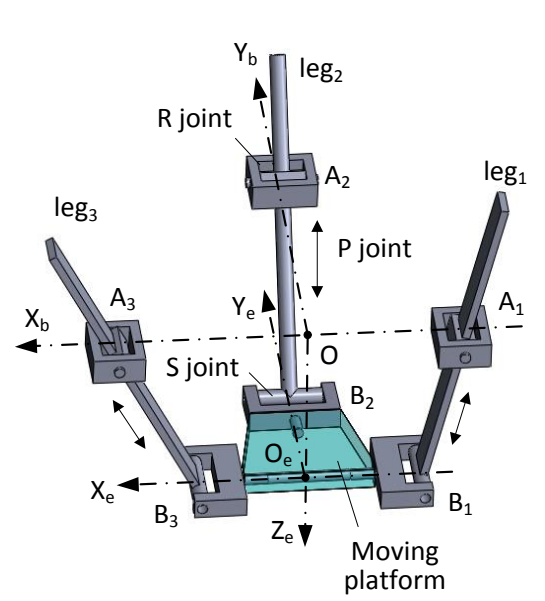

(b)

Fig. 1 Schematic diagram of (a) PAW (2RPU-1SPR) and (b) PAW-II (2RPU-1SPR)

For PAW, since point $O_{e}$ is always moves within the plane formed by points 
$A_{1}, B_{1}, B_{3}, A_{3}$, vector $\boldsymbol{O O}_{e}$ can be expressed as $\boldsymbol{O O}_{e}=\left[\begin{array}{lll}x_{e} & 0 & z_{e}\end{array}\right]^{T}$ in the base frame. Given the constraint offered by $\operatorname{leg}_{2}$, the determination of $x_{e}$ for the two PKMs are different. For PAW, as $X_{b}$ is perpendicular to the plane formed by points $A_{2}, B_{2}, O_{e}, x_{e}$ is obtained as follow.

$$
x_{e}=-\tan \alpha \cdot z_{e}
$$

For PAW-II, as the $\mathrm{R}$ joint axis of $\operatorname{leg}_{2}$ is perpendicular to $A_{2} B_{2}, x_{e}$ is calculated as

$$
x_{e}=\cos \beta \cdot \sin \alpha \cdot l_{2}
$$

Therefore, when $\alpha, \beta$ and $z_{e}$ are known, the actuators' displacements are obtained by

$$
q_{i}=\left|\boldsymbol{A}_{i} \boldsymbol{B}_{i}\right|=\left|\boldsymbol{O} \boldsymbol{O}_{e}+{ }_{o}^{o} R \cdot \boldsymbol{O}_{e} \boldsymbol{B}_{i}-\boldsymbol{O} \boldsymbol{A}_{i}\right|, \quad(i=1,2,3)
$$

As a result, both PAW and PAW-II have a unique inverse kinematic solution.

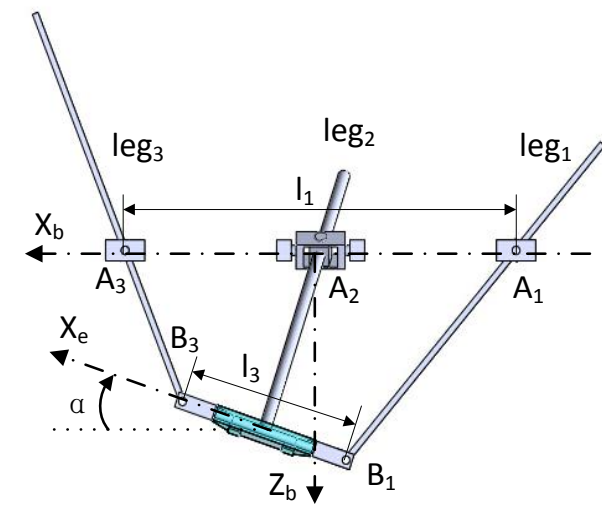

Front View

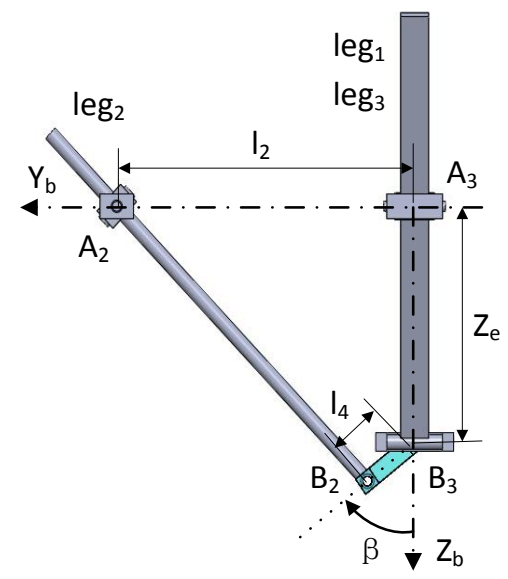

Right View

Fig. 2 Parameter description of PAW and PAW-II

\section{Kinematic performance}

Given the same dimensions to the two PKMs, as shown in Table 1, the workspace of PAW and PAW-II can be defined as the set of $\alpha, \beta$ and $z_{e}$ values, which can be obtained by examining the boundary formed by constraints via inverse 
kinematics.

Table 1. Nominal values of dimensional parameters

\begin{tabular}{cccc}
\hline$l_{1}$ & $l_{2}$ & $l_{3}$ & $l_{4}$ \\
\hline $1000 \mathrm{~mm}$ & $635 \mathrm{~mm}$ & $353 \mathrm{~mm}$ & $106 \mathrm{~mm}$ \\
\hline
\end{tabular}
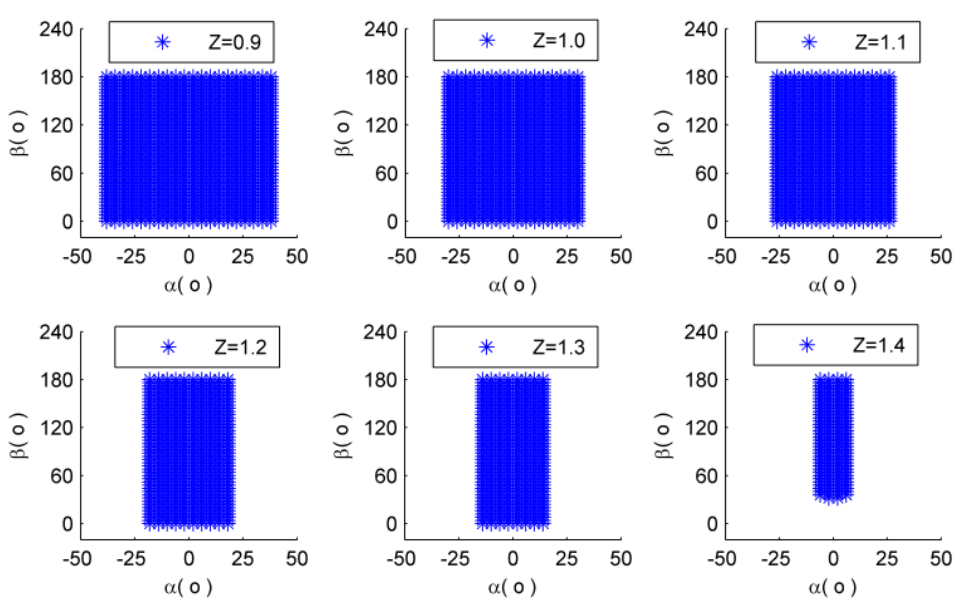

Fig. 3 Orientation workspace of PAW
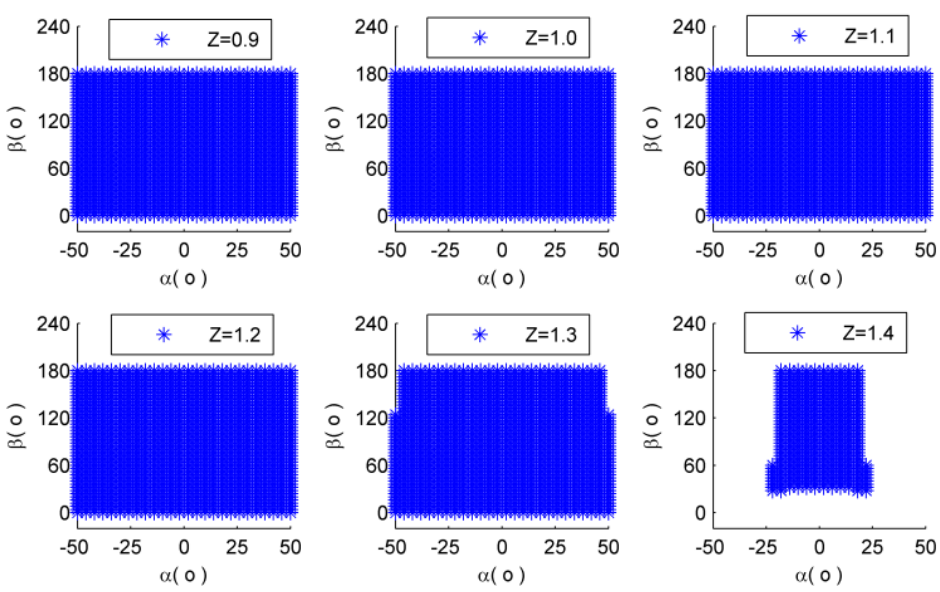

Fig. 4 Orientation workspace of PAW-II

For both PAW and PAW-II, the searching ranges of $\alpha, \beta$ and $z_{e}$ are set as $\left[-90^{\circ}, 90^{\circ}\right],\left[0,180^{\circ}\right]$ and $[0.9 \mathrm{~m}, 1.4 \mathrm{~m}]$. The constraints of three legs are $0.8 \mathrm{~m} \leq q_{1} \leq 1.5 \mathrm{~m}, 0.8 \mathrm{~m} \leq q_{3} \leq 1.5 \mathrm{~m} 0.9 \mathrm{~m} \leq q_{2} \leq 1.6 \mathrm{~m}$, and the rotating 
angles between $\mathrm{R}$ joints (on the base) and $Z_{b}$ axis are within $\left[20^{\circ}, 160^{\circ}\right]$.

The orientation workspaces for the two PKMs are shown in Fig. 3 and Fig. 4. It is obvious that the motion range of $\beta$ could reach from $0^{\circ}$ to $180^{\circ}$, while the motion range of $\alpha$ will become smaller with increasing $z_{e}$ value for both PAW and PAW-II. Examining their architectures, the motion range of $\alpha$ is constrained by the lengths of $\operatorname{leg}_{1}$ and $\operatorname{leg}_{3}$, as well as the location of the spherical joint in $\operatorname{leg}_{2}$. Since the spherical joint is on the moving platform rather than on the base, PAW-II has a much larger orientation capability in $\alpha$ than PAW. The orientation workspaces are symmetrical about $\alpha=0$. This is because of their symmetrical architecture about the $Y_{b} O Z_{b}$ plane at home position.

\section{Static performance}

Bonnemains et al. [19, 20] proposed energy based method for stiffness modeling, taking into account joint and leg deflections for over-constrained PKMs. In this paper, the same method is applied to PAW and PAW-II. Related stiffness characteristics of the two PKMs are listed in Table 2. By applying $1 \mathrm{KN}$ to point $O_{e}$ in $x, y, z$ directions respectively, the displacements in every direction is shown in Figures 4 - 9.

Table 2. Stiffness characteristics of PAW and PAW II PKMs

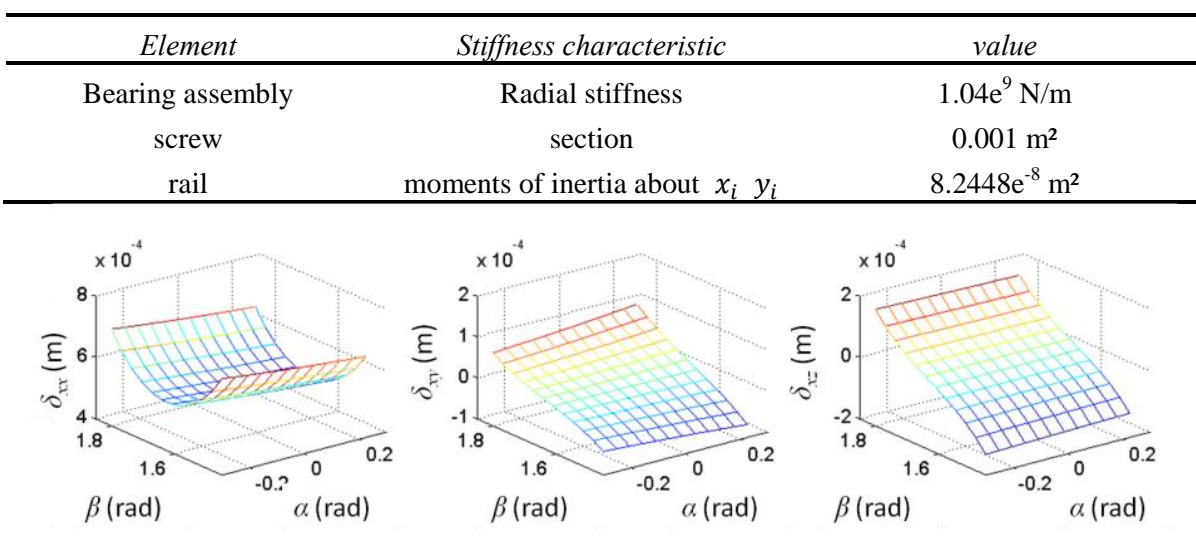

Fig. 5 Displacements of point $O_{e}$ for a $1 \mathrm{kN}$ effort in $\boldsymbol{x}$ direction and $z_{e}=1 \mathrm{~m}$ for PAW 

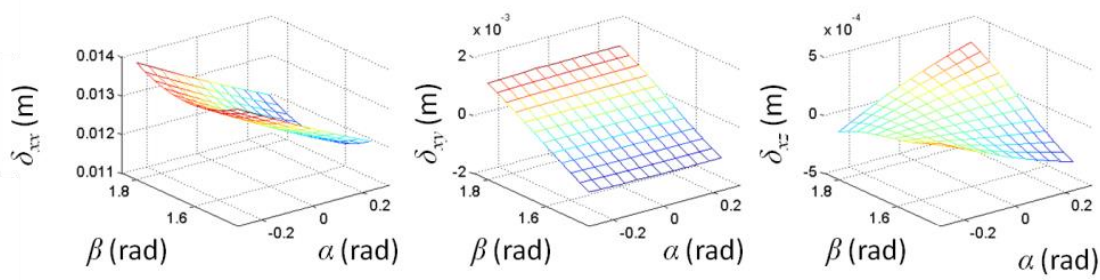

Fig. 6 Displacements of point $O_{e}$ for a $1 \mathrm{kN}$ effort in $\boldsymbol{x}$ direction and $z_{e}=1 \mathrm{~m}$ for PAW-II

Note that $\delta_{i j}$ represents displacement of point $O_{e}$ along axis $j$ for an effort applied in $i$ direction. Indeed, as PAW and PAW-II hold closed structures, an effort applied in a direction generates deflection in all the directions. As shown in Figures $5-6$, with an effort of $1 \mathrm{KN}$ exerted in $x$ direction, the biggest displacement are at the edge of workspace for both PAW and PAW-II. And the displacement of PAW is far smaller than that of PAW-II, which means that PAW is stiffer than PAW-II in $x$ direction. Note that displacements are not symmetrical due to orientation of the load in positive direction of $x$-axis.
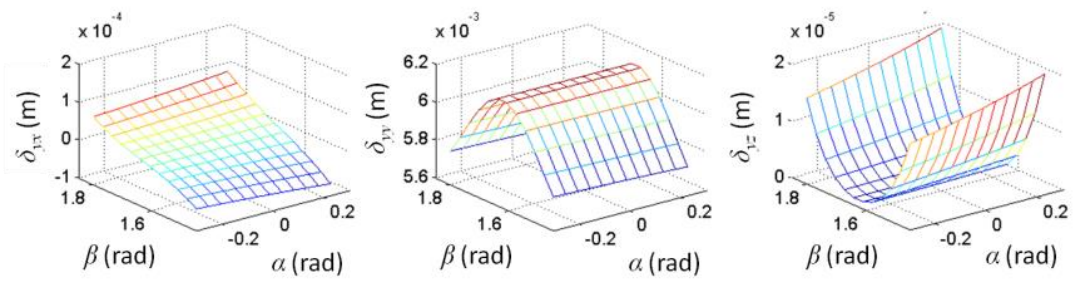

Fig. 7 Displacements of point $O_{e}$ for a $1 \mathrm{kN}$ effort in $y$ direction and $z_{e}=1 \mathrm{~m}$ for PAW
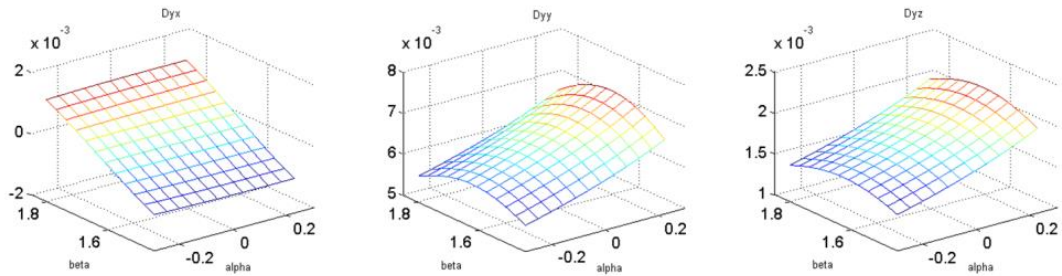

Fig. 8 Displacements of $O_{e}$ for a $1 \mathrm{kN}$ effort in $\boldsymbol{y}$ direction and $z_{e}=1 \mathrm{~m}$ for PAW-II 

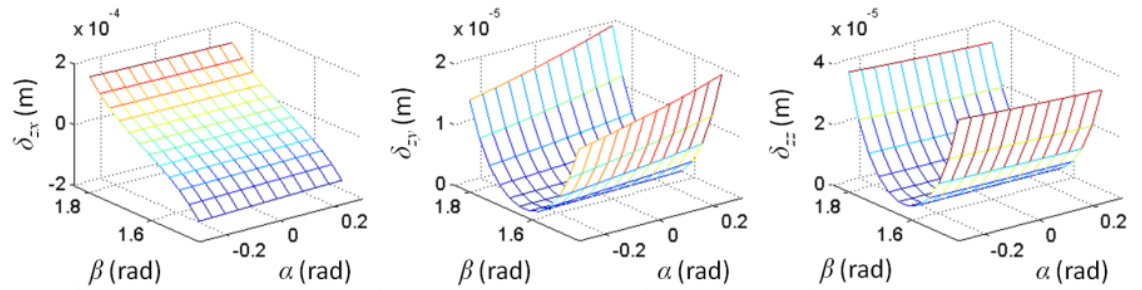

Fig. 9 Displacements of $O_{e}$ for a $1 \mathrm{kN}$ effort in $z$ direction and $z_{e}=1 \mathrm{~m}$ for PAW PM
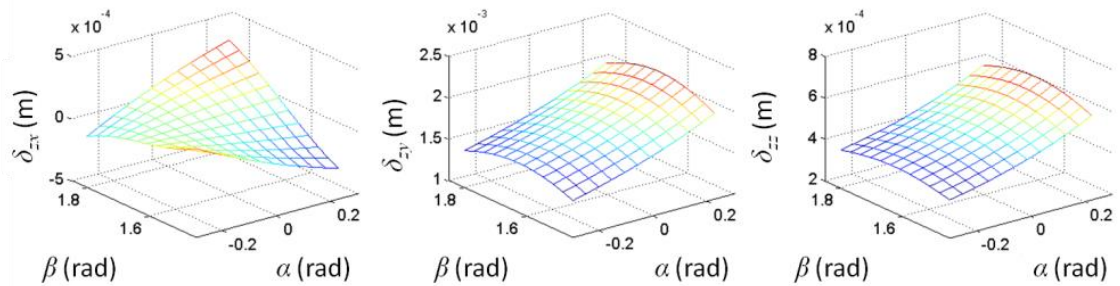

Fig. 10 Displacements of $O_{e}$ for a $1 \mathrm{kN}$ effort in $z$ direction and $z_{e}=1 \mathrm{~m}$ for PAW II PM

For the effort applied in $y$ direction (see Fig 7 and Fig. 8), both PAW and PAW-II show symmetrical distributions about $\beta=90^{\circ}$. For PAW, the maximum displacement is in the middle of the workspace while for PAW-II, it shows up at the workspace edge. Note that displacement of PAW along $y$ direction is slightly smaller, the stiffness of PAW is better than PAW-II.

In $z$ direction (see Fig 9 and Fig. 10), the biggest displacement of PAW is at the edges where $\beta$ reaches its limits. PAW-II gets its peak value when $\alpha$ is maximum. Comparing the two PKMs, displacements of PAW along $z$ direction is smaller than PAW-II. Still, PAW has better rigidity than PAW-II in $z$ direction.

Based on the above analysis, conclusion is drawn that PAW has overall better rigidity than PAW-II. This is mainly because the stiffness transmission from linear actuator to the moving platform is well transferred through an R joint for PAW, but not so well transferred through a spherical joint for PAW-II.

\section{Conclusion}

This paper first time conducts a comparative study on kinematic and static performance of two Exechon variants, PAW and PAW-II which represent two important families of 1T2R PKMs. The two PKMs show disparity merely in joint arrangement, where PAW has a SPR topology for $\operatorname{leg}_{2}$ while PAW-II has a RPS topology for $\operatorname{leg}_{2}$. It is found that the location of the joint arrangement has a dramatic effect on the workspace and stiffness performance of these PKMs. As a result, PAW has smaller workspace but better stiffness performance comparing with PAW-II. Both PAW and PAW-II have unique inverse kinematic solution, 
which will make ease for control. From this study, it is concluded that it is better to place a spherical joint on the base for achieving a better stiffness on the moving platform. For achieving better dexterity (larger orientation workspace), the spherical joint is better placed on the moving platform. Both two two PKMs have great potential for machine tool applications, depending on the specific operation requirements.

Acknowledgments The first author likes to acknowledge the funding support from Chinese Scholarship Council. The third author likes to acknowledge the funding support from the Engineering and Physical Science Research Council in United Kingdom under project no. EP/K004964/1.

\section{Reference}

1. Bi, Z. M. and Jin, Y.: Kinematic modeling of Exechon parallel kinematic machine, Robotics and Computer-Integrated Manufacturing, 27: 186-193 (2011)

2. Neumann, K.E.: System and method for controlling a robot, US Patent, No.6301525 (2001)

3. Xi, F. and Zhang, D. and Xu, Z. and Mechefske, C. M.: A comparative study on tripod units for machine tools, International Journal of Mechanism Tools and Manufacture, 43 (7): 721-730 (2003).

4. Chen, X. and Xie, F. G. and Liu, X. J.: A comparison study on motion/force transmissibility of two typical 3-DoF parallel manipulators: the sprint Z3 and A3 tool heads, International Journal of Advanced Robotic Systems, 11 (5) DOI: 10. 5772/57458 (2014)

5. Sun, T. and Song, Y. M. and Li, Y. G. and Liu, L. S.: Dimensional synthesis of a 3-DOF parallel manipulator based on dimensionally homogeneous Jacobian matrix, Science in China Series E: Technological Sciences, 53 (1): 168-174 (2010)

6. Refaat, S. and Herve, J. M. and Nahavandi, S. and Trinh, H.: Two-mode overconstrained three-DOFs rotational-translational linearmotor-based parallel-kinematics mechanism for machine tool applications, Robotica, 25(4): 461-466 (2007)

7. Li, Q.C. and Herve, J. M.: 1T2R Parallel Mechanisms Without Parasitic Motion, IEEE Transactions on Robotics, 26(3): 401-410 (2010)

8. Hunt, K.: Structural kinematics of in-parallel-actuated robot arms, ASME Journal of Mechanisms, Transaction, and Automation in Design, 105: 705-712 (1983)

9. $\mathrm{Li}, \mathrm{Y}$. and $\mathrm{Xu}, \mathrm{Q} .:$ Kinematic analysis of a 3-PRS parallel manipulator, Robotics and Computer-Integrated Manufacturing, 23(4): 395-408 (2007)

10. Li, Y. and Liu, H. T. and Zhao, X. M. and Huang, T. and Chetwynd, D. G.: Design of a 3-dof PKM module for large structural component machining, Mechanism and Machine Theory, 45(6): 941-954 (2010)

11. Rao, N. M. and Rao, K. M.: Dimensional synthesis of a spatial 3-RPS parallel manipulator for a prescribed range of motion of spherical joints, Mechanism and Machine Theory, 44(2): 477-486 (2009)

12. Sokolov, A. and Xirouchakis, P.: Dynamic analysis of a 3-dof parallel manipulator with R-P-S joint structure, Mechanism and Machine Theory, 42(5): 541-557 (2007)

13. Farhat, N. and Mata, V. and Page, A. and Valero, F.: Identification of dynamic parameters of a 3-DOF RPS parallel manipulator, Mechanism and Machine Theory, 43(1): 1-17 (2008)

14. Sun, T. and Song, Y. M. and Xu, L.: Separation of comprehensive geometrical errors of a 3-DoF parallel manipulator based on Jacobian matrix and its sensitivity analysis with Monte-Carlo method, 24(3): 1-8 (2011) 
15.Enferadi, J. and Tootoonchi, A. A.: Accuracy and stiffness analysis of a 3-RPS spherical parallel manipulator, Robotica, 29: 193-209 (2010)

16. Neumann, K.E.: The key to aerospace automation, In SAE aerospace Manufacturing and Automatic Fastening Conference and Exhibition, Michigan, USA, 2006-01-3144 (2006)

17. Jin, Y. and Bi, Z. and Gibson, R. and Mctoal, P. and Morgan, M. and Mcclory, C. and Higgins, C.: Kinematic analysis of a new Over-constraint parallel kinematic machine, Proceedings of IFToMM 2011 World Congress, Guanajuato, Mexico, A7-282 (2011)

18. Jin, Y. and Kong, X. W. and Higgins, C. and Price, M.: Kinematic design of a New parallel kinematic machine for aircraft wing assembly, $201210^{\text {th }}$ IEEE International Conference on Industrial Information, Beijing, China, 669-674 (2012)

19. Bonnemains, T. and Chanal, H. and Bouzgarrou, B. C. and Ray, P.: Stiffness computation and identification of parallel kinematic machine tools, Journal of Manufacturing Science and Engineering, 131: 041013-1-041013-7 (2009)

20. Bonnemains, T. and Chanal, H. and Bouzgarrou, B. C. and Ray, P.: Definition of a new static model of parallel kinematic machines: highlighting of overconstraint influence, 2008 IEEE/RSJ International Conference on Intelligent Robots and Systems Acropolis Convention Center, Nice, France, 2416-2421 (2008) 